\title{
Size-dependent decoherence of excitonic states in semiconductor microcrystallites
}

\author{
Yu-xi Liu, ${ }^{1}$ Adam Miranowicz, ${ }^{1,2,3}$ Şahin K. Özdemir, ${ }^{1,2}$ Masato Koashi, ${ }^{1,2}$ and Nobuyuki Imoto ${ }^{1,2,4}$ \\ ${ }^{1}$ The Graduate University for Advanced Studies (SOKENDAI), \\ Shonan Village, Hayama, Kanagawa 240-0193, Japan \\ ${ }^{2}$ CREST Research Team for Interacting Carrier Electronics, Hayama, Kanagawa 240-0193, Japan \\ ${ }^{3}$ Nonlinear Optics Division, Physics Institute, Adam Mickiewicz University, 61-614 Poznań, Poland \\ ${ }^{4}$ NTT Basic Research Laboratories, 3-1 Morinosato-Wakamiya, Atsugi, Kanagawa 243-0198, Japan
}

(Dated: January 25, 2019)

\begin{abstract}
The size-dependent decoherence of the exciton states resulting from the spontaneous emission is investigated in a semiconductor spherical microcrystallite under condition $a_{B} \ll R_{0} \leq \lambda$. In general, the larger size of the microcrystallite corresponds to the shorter coherence time. If the initial state is a superposition of two different excitonic coherent states, the coherence time depends on both the overlap of two excitonic coherent states and the size of the microcrystallite. When the system with fixed size is initially in the even or odd coherent states, the larger average number of the excitons corresponds to the faster decoherence. When the average number of the excitons is given, the bigger size of the microcrystallite corresponds to the faster decoherence. The decoherence of the exciton states for the materials GaAs and CdS is numerically studied by our theoretical analysis.
\end{abstract}

PACS numbers: 42.50.Fx, 71.35-y

The field of quantum computation and information processing is being extensively investigated due to possibility of finding solutions to some intractable problems on classical computers using the paradigm of quantum mechanics. Superposition principle of quantum mechanics forms the strong basis for the realization of the quantum computation and information processing schemes. The carrier of the quantum information is called as qubit which can take not only logical zero or one values as in its classical counterpart, but also their superposition. Any two-level system is suitable for the realization of qubits.

As low-dimensional semiconductor structures, quantum dots are very promising candidates for carrying quantum information because of their atom-like properties. The experimentalists have made great progress in the coherent observation and manipulation of states in systems of quantum dots 1, 2, 3], including demonstration of the quantum entanglement of excitons in a single dot [4] and a quantum dot molecule [5], or Rabi oscillations of excitons in single quantum dot [6]. The quantum gate realization based on the localized electron spins of quantum dots as qubits has been proposed [7]. A scheme of the controllable interactions between two distant quantum dot spins by combining the cavity quantum electrodynamics (QED) with electronic spin degrees of freedom in quantum dots was proposed [8], and the degree of quantum entanglement was further analyzed 9 ]. The reference [10] theoretically investigates the entanglement of excitonic states in the system of the optically driven coupled quantum dots and proposes a method to prepare maximally entangled Bell and GreenbergerHorne-Zeilinger states. An all optical implementation of quantum information processing with semiconductor macro atoms based on excitons was also proposed [11].

It is well known that pure quantum superposition states can hardly survive for a long time. Because the interaction of any system with the surrounding environment is unavoidable in a physical realization, the energy of the superposed state of the system is dissipated into environment or the relative phase of the superposed components is disturbed by the environment. The environment effect on the qubit defined by the exciton state has been investigated 12, 13, 14] in quantum dot with fixed size. However, in general, one can expect that increasing the size of a quantum dot results in stronger decoherence rates due to the increased overlap between the system and the environment.

In this report, the size-dependent decoherence is discussed in detail for a system of the semiconductor spherical microcrystallite (SSM) whose radius $R_{0}$ is smaller than the wavelength $\lambda$ of the relevant radiation field, but much larger than the Bohr radius $a_{B}$ of exciton in bulk semiconductor, i.e. $a_{B} \ll R_{0} \leq \lambda$. We assume that the excitation density is so low that the average number of excitons in the Bohr radius volume is no more than one. Thus the interactions between the excitons can be neglected. For the optically allowed lowest-energy excitons, the size-dependent Hamiltonian can be given under the rotating wave approximation as [15].

$$
\begin{aligned}
H & =\hbar \Omega b^{\dagger} b+\hbar \sum_{k} \omega_{k} a_{k}^{\dagger} a_{k} \\
& +\hbar \chi \sum_{k} \frac{\hat{e}_{k} \cdot \vec{\mu}_{c v}}{\sqrt{\hbar c k}}\left(b^{\dagger} a_{k}+b a_{k}^{\dagger}\right)
\end{aligned}
$$

with $\chi=\frac{4 \Omega R_{0}^{3 / 2}}{\sqrt{V \pi a_{B}^{3}}}$. Here $\hat{e}_{k}$ is a unit polarization vector of radiation field, $\vec{\mu}_{c v}$ is interband transition dipole moment, $V$ is volume of the microcrystallite, $k$ is wave vector of the radiation field, $a_{k}\left(a_{k}^{\dagger}\right)$ are the annihilation (creation) operators of radiation field with frequency $\omega_{k}$, 
and $b\left(b^{\dagger}\right)$ is the annihilation (creation) operator of the exciton with the transition energy $\hbar \Omega=E_{g}-E_{\text {exc }}^{b}+\frac{\hbar^{2} \pi^{2}}{2 M R_{0}^{2}}$ where $E_{g}$ is the energy gap between the conduction and valence bands, $E_{\text {exc }}^{b}$ is binding energy of the excitons and $M$ is the mass for the center-of-mass motion which is a sum of the effective masses $m_{e}$ and $m_{h}$ for the electron and hole, respectively, in the conduction and valence bands, i.e. $M=m_{e}+m_{h}$. The operators for both radiation field and excitons are bosons. The Hamiltonian (11) shows that both the exciton energy $\Omega$ and the coupling constants $\left(\chi \hat{e}_{k} \cdot \vec{\mu}_{c v}\right) / \sqrt{\hbar c k}$ between the exciton and radiation fields depend on the size $R_{0}$ of the microcrystallite, which means that the different sizes of the microcrystallites result in the different decay rates of the excitonic states.

In order to show how the different sizes of microcrystallites affect the coherence of the exciton states, we give the Heisenberg equations of motion for operators of the radiation field and excitons corresponding to Hamiltonian (11) as 16, 17]

$$
\begin{gathered}
\frac{\partial B(t)}{\partial t}=-i \chi \sum_{k} \frac{\hat{e}_{k} \cdot \vec{\mu}_{c v}}{\sqrt{\hbar c k}} A_{k}(t) e^{-i \omega_{k} t+i \Omega t} \\
\frac{\partial A_{k}(t)}{\partial t}=\quad-i \chi \frac{\hat{e}_{k} \cdot \vec{\mu}_{c v}}{\sqrt{\hbar c k}} B(t) e^{-i \Omega t+i \omega_{k} t}
\end{gathered}
$$

where we have applied rotating frame transformation to the exciton and radiation field variables with $b(t)=$ $B(t) e^{-i \Omega t}$ and $a_{k}(t)=A_{k}(t) e^{-i \omega_{k} t}$. Then we can obtain the formal solution of $A_{k}(t)$ as

$$
A_{k}(t)=A_{k}(0)-i \chi \frac{\hat{e}_{k} \cdot \vec{\mu}_{c v}}{\sqrt{\hbar c k}} \int_{0}^{t} B\left(t^{\prime}\right) e^{-i \Omega t^{\prime}+i \omega_{k} t^{\prime}} \mathrm{d} t^{\prime} .
$$

Substituting $A_{k}(t)$ in Eq.(2a), we get

$$
\begin{aligned}
\frac{\partial B(t)}{\partial t} & =-i \chi \sum_{k} \frac{\hat{e}_{k} \cdot \vec{\mu}_{c v}}{\sqrt{\hbar c k}} A_{k}(0) e^{-i \omega_{k} t+i \Omega t} \\
& -\int_{0}^{t} B\left(t^{\prime}\right) K\left(t-t^{\prime}\right) d t^{\prime}
\end{aligned}
$$

with the time-dependent kernel function

$$
K\left(t-t^{\prime}\right)=\chi^{2} \sum_{k} \frac{\left|\mu_{c v}\right|^{2} \cos ^{2} \theta}{\hbar c k} e^{-i\left(\Omega-\omega_{k}\right)\left(t^{\prime}-t\right)},
$$

where $\theta$ is the angle between the interband transition dipole moment $\vec{\mu}_{c v}$ and electric field polarization $\hat{e}_{k}$. Without loss of generality, we assume that the interband transition dipole moment $\vec{\mu}_{c v}$ of the optically allowed exciton is along the $z$ axis and radiation is isotropic. In order to give the time-dependent kernel function $K\left(t-t^{\prime}\right)$, we change the summation over $k$ into integral [15]

$$
\sum_{k} \rightarrow \frac{2 V}{(2 \pi)^{3}} \int_{0}^{\infty} \int_{0}^{2 \pi} \int_{0}^{\pi} \frac{\omega^{2}}{c^{3}} d \omega \sin \theta d \theta d \varphi
$$

where the integration is taken only over the volume $V$ of the microcrystallite, because the interband transition dipole moment $\vec{\mu}_{c v}$ vanishes outside the microcrystallite. The assumption of $R_{0} \leq \lambda$ makes the polariton effect negligible implying the rapid radiation of excitons due to the breakdown of the translation symmetry. Consequently, we can consider that the radiation light intensity of microcrystallite is going to be centered at the transition frequency $\Omega$, which is known as the Weisskopf-Wigner approximation [16]. Then the kernel function is found as

$$
K\left(t-t^{\prime}\right)=32 \pi\left(\frac{R_{0}}{a_{B}}\right)^{3} \gamma_{s} \delta\left(t^{\prime}-t\right)
$$

with $\gamma_{s}=\frac{4\left|\mu_{c v}\right|^{2} \Omega^{3}}{3 \hbar(2 \pi c)^{3}}$ depending on the size of microcrystallite. Replacing the kernel function of Eq. (7) in Eq. (4) and taking the Laplace transform, the exciton operator $B(t)$ is found as

$$
B(t)=B(0) e^{-32 \pi\left(\frac{R_{0}}{a_{B}}\right)^{3} \gamma_{s} t}-i \sum_{k} u_{k}(t) A_{k}(0),
$$

$u_{k}(t)$ is given as

$$
u_{k}(t)=\frac{\chi \hat{e}_{k} \cdot \vec{\mu}_{c v}}{\sqrt{\hbar c k}} \frac{\exp \left(-64 \pi \frac{R_{0}^{3}}{a^{3}} \gamma_{s} t\right)-\exp \left[-i\left(\omega_{k}-\Omega\right) t\right]}{-64 \pi \frac{R_{0}^{3}}{a^{3}} \gamma_{s}+i\left(\omega_{k}-\Omega\right)} .
$$

From Eq. (8), it can be found that the spontaneous emission rate of exciton is $64 \pi\left(R_{0} / a_{B}\right)^{3}$ times higher than that of an atom which has the transition frequency $\Omega$ and the transition dipole moment $\vec{\mu}_{c v}$ [15. This increase in the spontaneous emission rate can be explained by the fact that the exciton is coherently excited over the whole quantum microcrystallite. Thus coherent excitation results in a coherent transition dipole moment and a superradiant character. It is obvious that the information, which is carried by the state of the exciton, is lost with the time evolution because of the energy dissipation due to spontaneous emission of the exciton. The loss rate of the information depends on the factor $32 \pi\left(R_{0} / a_{B}\right)^{3} \gamma_{s}$.

Now lets analyze how the size-dependent decoherence affect a qubit defined in the computational basis of the vacuum state $|0\rangle$ and single-exciton state $|1\rangle$. If the exciton system is initially in a qubit state $\alpha|0\rangle+\beta|1\rangle$ with $|\alpha|^{2}+|\beta|^{2}=1$, and the radiation field is initially in the multimode vacuum state $\prod_{k}|0\rangle_{k}$, after an evolution time of $t$, the state of the system will become [12],

$$
\begin{aligned}
& |\psi(t)\rangle=U(t)[\alpha|0\rangle+\beta|1\rangle] \otimes \prod_{k}|0\rangle_{k} \\
& =\alpha|0\rangle \otimes \prod_{k}|0\rangle_{k}+\beta U(t) b^{\dagger}|0\rangle \otimes \prod_{k}|0\rangle_{k} \\
& =\left[\alpha|0\rangle+\beta e^{\left\{-32 \pi\left(\frac{R_{0}}{a_{B}}\right)^{3} \gamma_{s} t-i \Omega t\right\}}|1\rangle\right] \otimes \prod_{k}|0\rangle_{k} \\
& +\beta|0\rangle \otimes \sum_{k^{\prime}} u_{k^{\prime}}(t) e^{-i\left(\Omega+\omega_{k^{\prime}}\right) t}|1\rangle_{k^{\prime}} \otimes \prod_{k \neq k^{\prime}}|0\rangle_{k},
\end{aligned}
$$




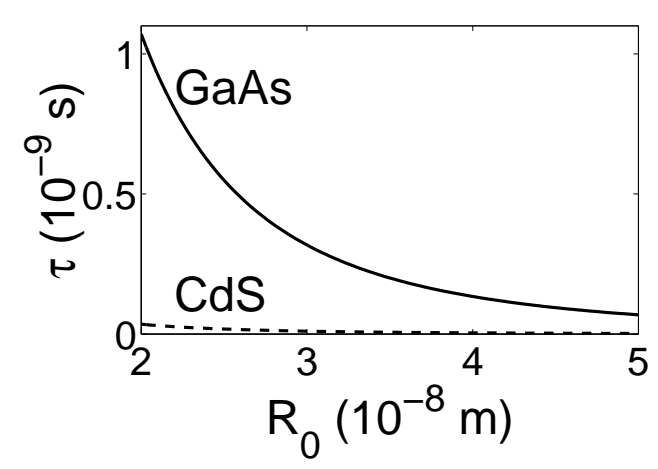

FIG. 1: Characteristic time $\tau$ as a function of size $200 \AA<$ $R_{0}<500 \AA$ for the CdS and GaAs microcrystallites when the systems are initially in the superposition of the vacuum and single-exciton states.

where the properties of the time evolution operator $U^{\dagger}(t) b U(t)=b(t)$ and $U(t)|0\rangle=0$ with $U(t)=e^{-i H t / \hbar}$ are applied. Because we are interested in the exciton system, the multimode radiation field degrees of freedom are traced out and the reduced density operator of the exciton system is found as

$$
\begin{aligned}
\rho(t) & =\left(1-\eta(t)|\beta|^{2}\right)|0\rangle\left\langle 0\left|+\alpha \beta^{*} F(t) e^{i \Omega t}\right| 0\right\rangle\langle 1| \\
& +\alpha^{*} \beta F(t) e^{-i \Omega t}|1\rangle\left\langle\left. 0|+\eta(t)| \beta\right|^{2} \mid 1\right\rangle\langle 1|
\end{aligned}
$$

with $\eta(t)=\exp \left\{-64 \pi\left(\frac{R_{0}}{a_{B}}\right)^{3} \gamma_{s} t\right\}$. The slowly varying time-dependent factor $F(t)$ of the off-diagonal elements in Eq.(11), which can be written as

$$
F(t)=\exp \left\{-32 \pi\left(\frac{R_{0}}{a_{B}}\right)^{3} \gamma_{s} t\right\}=\exp (-t / \tau)
$$

is used to characterize the coherence of the superposed excitonic state $\alpha|0\rangle+\beta|1\rangle$. The characteristic time $\tau$ of the decoherence is given as,

$$
\tau=\frac{3 \hbar^{4} \pi^{2} c^{3} a_{B}^{3} M^{3} R_{0}^{3}}{2\left|\mu_{c v}\right|^{2}\left[2 M R_{0}^{2}\left(E_{g}-E_{e x c}^{b}\right)+\hbar^{2} \pi^{2}\right]^{3}} .
$$

To give a better insight to the problem, we give some numerical values for the chosen materials, CdS and GaAs. The following characteristic parameters for those materials are taken from references [15, 18]. For CdS, $E_{g}=2.583 \mathrm{eV}, E_{\text {exc }}^{b}=30 \mathrm{meV}, a_{B}=30 \AA, \frac{\left|\mu_{c v}\right|^{2}}{\left(\epsilon_{0} a_{B}^{3}\right)}=0.25$ meV with the static dielectric constant of the bulk crystal $\epsilon_{0}=8, m_{e}=0.25 m_{0}$ and $m_{h}=1.6 m_{0}$ where $m_{0}$ is the rest mass of electron. For GaAs, $E_{g}=1.52 \mathrm{meV}$, $E_{e x c}^{b}=5 \mathrm{meV}, a_{B}=100 \AA, \frac{\left|\mu_{c v}\right|^{2}}{\left(\epsilon_{0} a_{B}^{3}\right)}=0.025 \mathrm{meV}$ with $\epsilon_{0}=12.53, m_{e}=0.0665 m_{0}$ and $m_{h}=0.45 m_{0}$. The area of the microcrystallite size is taken as $200 \AA \leq R_{0} \leq 500 \AA$ in Fig. 11 In this range, the wavelength is around 5000 $\AA$ for $\mathrm{CdS}$, and $8000 \AA$ for GaAs, thus the condition $a_{B} \ll R_{0} \leq \lambda$ is satisfied.
In order to show how the characteristic time $\tau$ changes with the size $R_{0}$, we give Fig. 11 It is seen that a larger size of the microcrystallite corresponds to a shorter characteristic time $\tau$. So the larger is the size of microcrystallite, the faster is the decoherence of the superposition of two exciton states for a given material. But the exciton state for different materials have the different decoherence times even if the size of the microcrystallites are the same. This is due to the different couplings of the systems with the radiation field. Comparing the decoherence characteristic time of CdS and GaAs in Fig. 1 we find that the coherence time of the exciton state for GaAs is longer than that of CdS for a given size. This result shows the importance of material choice in obtaining longer coherence time.

Here, we consider another kind of qubit state formed by the superposition of coherent states, for example, we can define the even coherent state $\mathcal{N}_{+}[|\alpha\rangle+|-\alpha\rangle]$ as the logic zero state $|0\rangle_{L}$ and the odd coherent state $\mathcal{N}_{-}[|\alpha\rangle-|-\alpha\rangle]$ as the logic one state $|1\rangle_{L}$ where $\mathcal{N}_{ \pm}=$ $\left(2 \pm 2 e^{-2|\alpha|^{2}}\right)^{-1 / 2}$ and $|\alpha\rangle$ is an eigenstate of the bosonic operator. These states can be prepared by the photon states in cavity quantum electrodynamics and by motional states of trapped ions [19]. Such logical qubit encoding can be used as the correction of the spontaneous emission errors 20]. We first assume that the exciton system is initially in a superposition state of two coherent states $\left|\alpha_{1}\right\rangle$ and $\left|\alpha_{2}\right\rangle$ of the exciton annihilation operator

$$
|\psi\rangle=C\left|\alpha_{1}\right\rangle+D\left|\alpha_{2}\right\rangle,
$$

and the radiation field is in the multimode vacuum state $\prod_{k}|0\rangle_{k}$. By using the same approach as reference [17] and Eq.(8), we can find that the time-dependent reduced density operator for the exciton system is

$$
\begin{aligned}
\rho(t) & =|C|^{2}\left|u(t) \alpha_{1}\right\rangle\left\langle u(t) \alpha_{1}\left|+C D^{*} F(t)\right| u(t) \alpha_{1}\right\rangle\left\langle u(t) \alpha_{2}\right| \\
& +C^{*} D F^{*}(t)\left|u(t) \alpha_{2}\right\rangle\left\langle u(t) \alpha_{1}\right| \\
& +|D|^{2}\left|u(t) \alpha_{2}\right\rangle\left\langle u(t) \alpha_{2}\right|
\end{aligned}
$$

where $u(t)=\exp \left\{-32 \pi\left(\frac{R_{0}}{a_{B}}\right)^{3} \gamma_{s} t-i \Omega t\right\}$ and the timedependent decoherence factor is

$$
\begin{aligned}
F(t) & =\exp \left\{\left[-\frac{\left|\alpha_{1}\right|^{2}}{2}-\frac{\left|\alpha_{2}\right|^{2}}{2}+\alpha_{1} \alpha_{2}^{*}\right]\right. \\
& \left.\times\left[1-\exp \left\{-32 \pi\left(\frac{R_{0}}{a_{B}}\right)^{3} \gamma_{s} t\right\}\right]\right\} .
\end{aligned}
$$

It is understood that the decoherence of the superposition state of two coherent states is determined by both the properties of the microcrystallite and the overlap between two coherent states. Due to the existence of the vacuum state component in the excitonic coherent state, it is also observed that the decoherence factor $F(t)$ does not tend to zero in the long time limit $t \rightarrow \infty$.

For the qubit states which are defined by the even and odd coherent states (also referred as the Schrödinger 

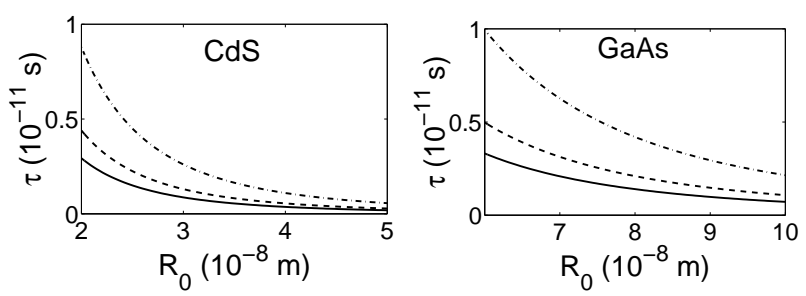

FIG. 2: Characteristic time $\tau$ as a function of size $200 \AA<$ $R_{0}<500 \AA$ for $\mathrm{CdS}$ and $600 \AA<R_{0}<1000 \AA$ for GaAs microcrystallites for different average numbers of excitons: $|\alpha|^{2}=2$ (dot-dashed curves), $|\alpha|^{2}=4$ (dashed curves), $|\alpha|^{2}=6$ (solid curves) when the systems are initially in the even or odd coherent states

cats), the decoherence factor is

$$
F(t)=\exp \left\{-2|\alpha|^{2}\left[1-e^{\left\{-32 \pi\left(\frac{R_{0}}{a_{B}}\right)^{3} \gamma_{s} t\right\}}\right]\right\} .
$$

For the behavior of the short time $32 \pi\left(\frac{R_{0}}{a_{B}}\right)^{3} \gamma_{s} t \ll 1$, the characteristic time $\tau$ of the decoherence for the superposed excitonic coherent states is approximately $1 /\left(2|\alpha|^{2}\right)$ times of that in Eq. (13). In order to discuss how the characteristic time depends on the size and the average number of the excitons in detail. We plot Fig. 2 with different average numbers of the excitons in ranges $200 \AA \leq R_{0} \leq 500 \AA$ for $\mathrm{CdS}$ and $600 \AA \leq R_{0} \leq 1000$ $\AA$ for GaAs, in which the conditions of the bosonic descriptions of excitons and $a_{B} \ll R_{0} \leq \lambda$ are still valid. It is found that the larger average number of the excitons corresponds to the shorter characteristic time when the size of the microcrystallite is fixed, and the larger size of the microcrystallite corresponds to the faster decoherence when the average number of the excitons is fixed.

In summary, we have studied the size-dependent decoherence for the semiconductor microcrystallites. It is found that microcrystallites with larger sizes have shorter coherence time of the exciton states. If the system is initially in the superposition of two different excitonic coherent states, the coherence characteristic time depends on both the sizes of the microcrystallites and the overlap between the two coherent states. A numerical study of the decoherence of the exciton states for the materials GaAs and CdS is carried out based on our theoretical analysis. This numerical analysis clearly shows the importance of material choice on the decoherence characteristics of microcrystallites. It also should be pointed out that our study cannot simply be generalized to the small quantum dot whose effective radius $R_{0}$ is smaller than the Bohr radius $a_{B}$ in the bulk semiconductor when there exist many excitons in a quantum dot. Because the Pauli principle prohibits the two or more excitons from occupying the same energy state in the small quantum dot. The details need to be studied further.

Yu-xi Liu is supported by Japan Society for the Promotion of Science (JSPS).

[1] N. H. Bonadeo et al., Science 282, 1473 (1998).

[2] T. H. Oosterkamp et al., Nature 385, 873 (1998).

[3] T. Fujisawa et al., Nature 419, 278 (2002).

[4] G. Chen et al., Science 289, 1906 (2000).

[5] M. Bayer et al., Science 291, 451 (2001).

[6] T. H. Stievater et al., Phys. Rev. Lett. 87, 133603 (2001); H. Kamada et al., ibid. 87, 246401 (2001).

[7] A. Barenco et al., Phys. Rev. Lett. 74, 4083 (1995); D. Loss and P. DiVincenzo, Phys. Rev. A 57, 120 (1998); Xuedong Hu and S. Das Sarma, ibid. 61, 062301 (2000); 64, 042312 (2001).

[8] A. Imamoğlu et al., Phys. Rev. Lett. 83, 4204 (1999).

[9] A. Miranowicz et al., Phys. Rev. A 65, 062321 (2002).

[10] L. Quiroga et al., Phys. Rev. Lett. 83, 2270 (1999); J. H. Reina et al., Phys. Rev. A 62, 012305 (2000).

[11] E. Biolatti et al., Phys. Rev. Lett. 85, 5647 (2000); Phys. Rev. B 65, 075306 (2002).

[12] Yu-xi Liu et al., Phys. Rev. A 65, 042326 (2002); Yu-xi Liu et al., ibid. 66, 062309 (2002).

[13] F. J. Rodriguez et al., Physica Status Solidi (a) 178, 403 (2000).

[14] P. Zanardi and F. Rossi, Phys. Rev. Lett. 81, 4752 (1998).

[15] E. Hanamura, Phys. Rev. B 37, 1273 (1988); ibid. 38, 1228 (1988).

[16] M. O. Scully and M. S. Zubairy, Quantum Optics (Cambridge Univeristy Press, Cambridge, 1997).

[17] Yu-xi Liu et al., Phys. Rev. A 63, 033816 (2001).

[18] D. B. Tran Thoai et al., Phys. Rev. B 42, 11261 (1990); ibid. 42, 5906 (1990).

[19] M. Brune et al., Phys. Rev. Lett. 77, 4887 (1996); C. Monroe et al., Science 272, 1131 (1996).

[20] P. T. Cochrane et al., Phys. Rev. A 59, 2631 (1999). 